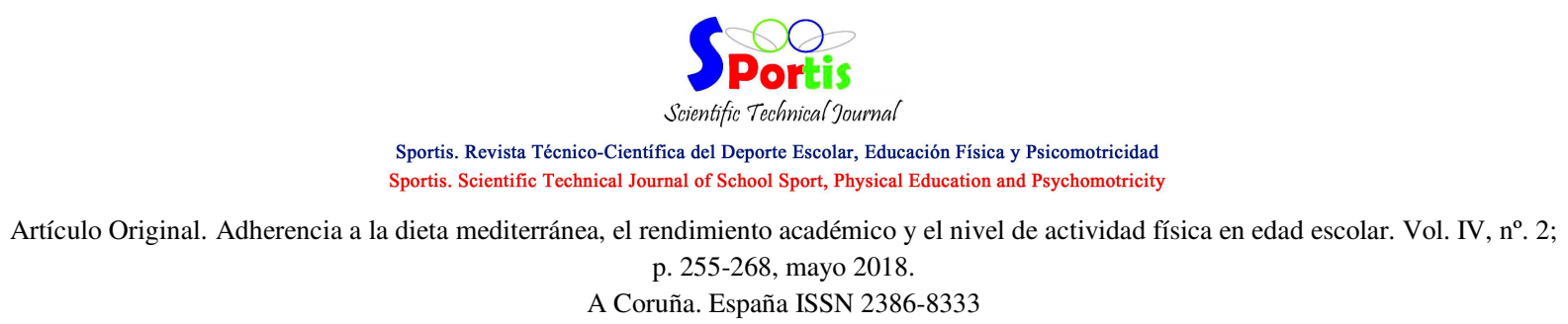

\title{
Adherencia a la dieta mediterránea, el rendimiento académico y el nivel de actividad física en edad escolar
}

\section{Mediterranean diet, academic performance and physical activity level in scholars}

\author{
Rosa $\mathrm{M}^{\mathrm{a}}$ Alfonso Rosa ${ }^{1}$; Francisco Álvarez Barbosa ${ }^{2}$ J Jesús del Pozo Cruz ${ }^{1}$ \\ ${ }^{1}$ Facultad Ciencias de la Educación. Universidad de Sevilla \\ ${ }^{2}$ Departamento de Actividad Física y Deporte. Fundación San Pablo CEU \\ Contacto: roalrosa@us.es
}

Cronograma editorial: Artículo recibido: 18/10/2017 Aceptado: 7/03/2018 Publicado: 01/05/2018

DOI: https://doi.org/10.17979/sportis.2018.4.2.3108

\section{Resumen}

El objetivo del estudio fue describir y establecer las posibles relaciones entre la adherencia a la dieta mediterránea, rendimiento académico y nivel de actividad física en alumnos de Educación Primaria pertenecientes a un centro de la provincia de Sevilla. Se usó un diseño de corte transversal-observacional. La muestra estuvo compuesta por 50 sujetos (26 niños y 24 niñas) con edades comprendidas entre los 9 y 10 años. Para la recogida de datos se administraron un cuestionario autorregistro, test Kidmed, cuestionario IPAQ-A y las calificaciones de las asignaturas comunes. Los resultados muestran que el 50\% de los sujetos tuvieron una adherencia a la dieta mediterránea óptima, frente al $10 \%$ de los sujetos que tuvieron una adherencia baja. Por otro lado, el $40 \%$ de los sujetos reflejó la necesidad de mejorar el patrón alimentario para adecuarlo al modelo mediterráneo. Respecto al nivel de actividad física, el $60 \%$ de los sujetos eran inactivos o muy pocos activos, frente al $25 \%$ que fueron muy activos. Por último, se halló una asociación entre la adherencia a la dieta mediterránea y las calificaciones obtenidas en las diferentes asignaturas [Educación Artística (Coeficiente contingencia $=, 442, \mathrm{p}<0,05)$, Matemáticas (Coeficiente contingencia $=, 484$, $\mathrm{p}<0,05)$ y Ciencias Sociales (Coeficiente contingencia $=, 490, \mathrm{p}<0,05)]$. En conclusión, podemos decir que el $50 \%$ de los sujetos tiene que mejorar su patrón alimentario, que tan solo el 14\% realiza la actividad física recomendada y que existió asociación entre la adherencia a la dieta mediterránea y las calificaciones obtenidas en Educación Artística, Matemáticas y Ciencias Sociales.

\section{Palabras clave}

Dieta mediterránea; actividad física; rendimiento académico; escolares.

Para citar este artículo utilice la siguiente referencia: Alfonso Rosa, R. Mạ.;Álvarez-Barbosa, F.; Del Pozo Cruz, J. (2018). Adherencia a la dieta mediterránea, el rendimiento académico y el nivel de actividad física en edad escolar. Sportis Sci J, 4 (2), 255-268. DOI:https://doi.org/10.17979/sportis.2018.4.2.3108 


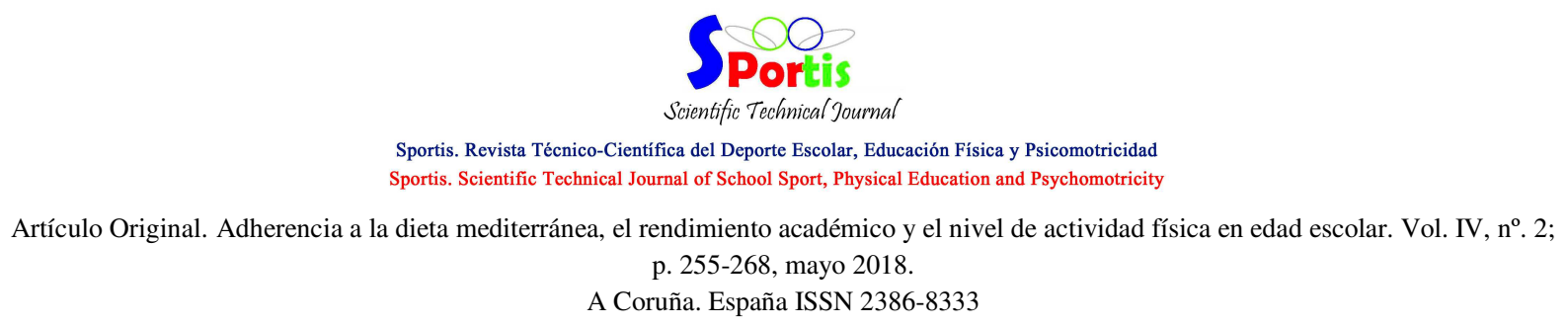

\begin{abstract}
The aims of the study were to describe and establish the possible relationships between adherence to the mediterranean diet, academic performance, and level of physical activity in Primary Education students from a center in the province of Seville. A cross-observational cross-sectional design was used. The sample consisted of 50 subjects ( 26 boys and 24 girls) aged between 9 and 10 years. For data collection, a self-register questionnaire, Kidmed test, IPAQ-A questionnaire and the grades of the common subjects were used. The results show that $50 \%$ of the subjects applied adherence to the optimal mediterranean diet, compared to $10 \%$ of subjects who applied a low adherence. On the other hand, $40 \%$ of the subjects showed the need to improve the dietary pattern to adapt to the mediterranean model. Regarding the level of physical activity, $60 \%$ of subjects were inactive or very few active, compared to $25 \%$ who were very active. Finally, an association between adherence to the mediterranean diet and the grades obtained in the different subjects (Contingency Coefficient $=.442, p<0.05$ ), Mathematics (Contingency Coefficient $=.484, \mathrm{p}<0.05$ ) and Social Sciences (Contingency Coefficient $=.490, \mathrm{p}<0.05)]$. In conclusion, I can say that $50 \%$ of the subjects should improve their dietary pattern, that only $14 \%$ do the recommended physical activity and that there was an association between the addition to the mediterranean diet and the grades obtained in Artistic Education, Mathematics and Social Sciences.
\end{abstract}

\title{
Keywords
}

Mediterranean diet; physical activity; academic performance; scholars.

\section{Introducción}

En la actualidad, el incremento del sobrepeso y la obesidad infantil han sido dramáticos (AECOSAN, 2016; OMS, 2014), asociándose a un amplio grupo de complicaciones para la salud, así como a un mayor riesgo de enfermedad cardiovascular y a una muerte prematura en la edad adulta (Estruch et al., 2013; Palmer et al., 2009). La reducción en los niveles de practica de actividad física y el abandono de la dieta mediterránea son dos de los factores fundamentales que están provocando este drástico aumento (Owen, Sparling, Healy, Dunstan, \& Matthews, 2010; Tognon et al., 2014). 


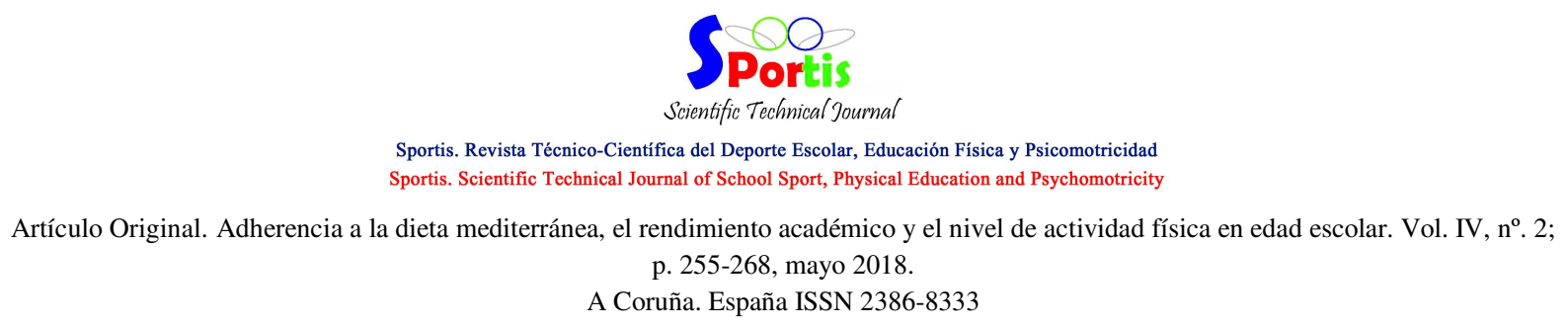

En este sentido, la infancia es considerada un periodo clave para la consolidación de la personalidad, así como la adquisición un estilo de vida adecuado (Macias, Gordillo, \& Camacho, 2012). Es una evidencia, que la adquisición de patrones de vida durante la infancia perdurará durante toda la vida (Macias et al., 2012). Preocupados por la repercusión que la adquisición de hábitos de vida saludable puede tener en la salud y calidad de vida de estos infantes, son varios los estudios que se han ido desarrollando sobre las conductas de salud en este grupo de edad (Costarelli, Koretsi, \& Georgitsogianni, 2013; Molinero et al., 2010).

Algunos estudios destacan el abandono progresivo de la dieta mediterránea, siendo éste más acentuado en zonas urbanizadas frente a las poblaciones rurales (Farajian et al., 2011; Lazarou \& Kalavana, 2009), aunque en la actualizad no existen conclusiones definitivas al respecto (Karlén, Lowert, Chatziarsenis, Fälth-Magnusson, \& Faresjö, n.d.; Serra-Majem, Ribas, et al., 2004).

En líneas generales, la dieta mediterránea incluye no solo un patrón alimenticio conocido, sino también social, así como aspectos gastronómicos que caracterizan un cierto estilo de vida. Combina alimentos de la agricultura local, recetas y métodos de cocción tradicionales de cada zona geográfica, junto con la práctica de actividad física regular (Donini, Serra-Majem, Bulló, Gil, \& Salas-Salvadó, 2015). Esta dieta se caracteriza por ser pobre en grasas saturadas y rica en antioxidantes, basada principalmente en el consumo de verduras, frutas, hortalizas, legumbres, pescado, frutos secos y aceite de oliva (Castro-Quezada, Román-Viñas, \& SerraMajem, 2014). Varios estudios muestran como este modelo de dieta juega un papel preventivo en la aparición de enfermedades cardiovasculares, metabólicas, oncológicas, entre otras (Bulló, Lamuela-Raventós, \& Salas-Salvadó, 2011; Nadtochiy \& Redman, 2011).

Como resultado de ello, en los últimos años se ha incrementado el número de estudios centrados en la influencia de la dieta mediterránea en esta población. Además, existe gran interés en determinar la relación de los hábitos alimenticios de esta población con otros componentes de su estilo de vida como la actividad física. En general, se ha demostrado que los jóvenes con una alta adherencia a esta dieta tienen mayores beneficios físicos y muestran mayores niveles de calidad de vida relacionada con la salud (Costarelli et al., 2013).

Para citar este artículo utilice la siguiente referencia: Alfonso Rosa, R. Ma.;Álvarez-Barbosa, F.; Del Pozo Cruz, J. (2018). Adherencia a la dieta mediterránea, el rendimiento académico y el nivel de actividad física en edad escolar. Sportis Sci J, 4 (2), 255-268. DOI:https://doi.org/10.17979/sportis.2018.4.2.3108 


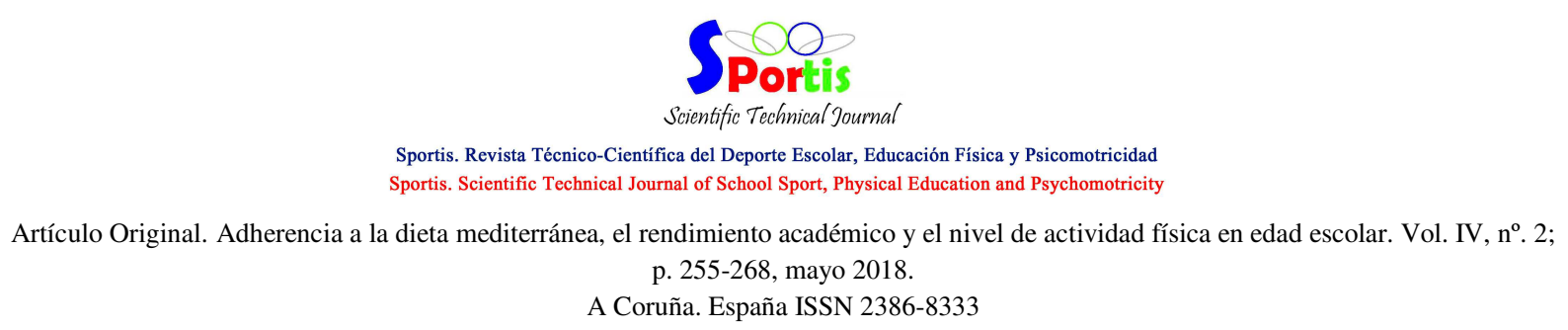

Por otro lado, otro de los factores que marca la infancia de los escolares y su relación con el entorno, no solo en los centros educativos si no en el seno de la familia, es el rendimiento académico obtenido durante la etapa de escolarización. Así, Álvarez-Bueno et al., (2017) muestran que la actividad física, en concreto la Educación Física, mejora el comportamiento en el aula y beneficia varios aspectos del rendimiento académico, especialmente las habilidades relacionadas con las matemáticas y la lectura.

Por ello, el objetivo del estudio fue describir y establecer las posibles relaciones entre la adherencia a la dieta mediterránea, rendimiento académico y nivel de actividad física en alumnos de educación primaria pertenecientes a un centro de la provincia de Sevilla.

\section{Material y Método}

\section{Diseño y participantes}

En el presente estudio se usó un diseño de corte transversal-observacional. La muestra estuvo compuesta por 50 sujetos (26 niños y 24 niñas) con edades comprendidas entre los 9 y 10 años, pertenecientes a un centro de Educación Primaria de la provincia de Sevilla. El criterio de inclusión fue: pertenecer a $3^{\circ}$ de Educación Primaria. Como criterio de exclusión se estableció: alumnos que tuvieran algún problema cognitivo que acepte al correcto entendimiento de los cuestionarios.

Para su desarrollo el padre, madre o tutor legal fueron informados del propósito del estudio tanto de forma escrita como oral, y firmaron un formulario de consentimiento informado. Además, dicha investigación fue llevada a cabo bajo las consideraciones éticas para el estudio con humanos recogida en la Declaración de Helsinki (2008).

\section{Medidas e instrumentos}

A continuación, se describen los diferentes instrumentos utilizados para la recogida de información:

- $\quad$ Autorregistro: a través de esta primera parte se recogieron variables sociodemográficas (edad, género....).

Para citar este artículo utilice la siguiente referencia: Alfonso Rosa, R. Ma.;Álvarez-Barbosa, F.; Del Pozo Cruz, J. (2018). Adherencia a la dieta mediterránea, el rendimiento académico y el nivel de actividad física en edad escolar. Sportis Sci J, 4 (2), 255-268. DOI:https://doi.org/10.17979/sportis.2018.4.2.3108 


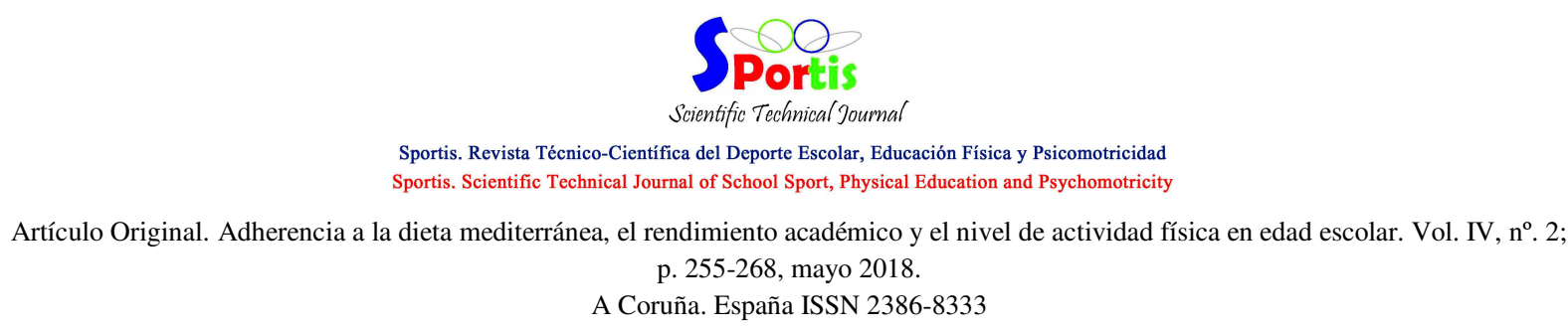

Adherencia a la dieta mediterránea: para evaluar la adherencia a la dieta mediterránea se usó el test KIDMED (Serra-Majem, Ribas, et al., 2004), el cual se compone de 16 preguntas las cuales deben responderse afirmativa o negativamente (si/no). Las respuestas afirmativas en las 12 preguntas que representan un aspecto positivo en relación con la dieta mediterránea suman 1 punto, mientras que las respuestas afirmativas en las preguntas (son 4) que representan una connotación negativa restan un punto. La puntuación total da lugar a un índice KIDMED, que permite clasificar en tres grupos el grado de adherencia a la dieta mediterránea:

- $\quad$ De 0 a 3: dieta de muy baja calidad (adherencia a la dieta mediterránea baja).

- $\quad$ De 4 a 7: necesidad de mejora en el patrón alimentario para adecuarlo al modelo mediterráneo (adherencia media).

- $\quad$ De 8 a 12: dieta mediterránea óptima (adherencia alta).

- Rendimiento académico: para conocer el rendimiento académico de los alumnos se recogieron las calificaciones de las asignaturas comunes (Lengua, Matemáticas, Educación Física, Educación Musical, Educación Plástica y Visual, Inglés, Ciencias Sociales y Ciencias Naturales). La valoración académica se codificó de 1 a 5 donde 5 es la máxima nota (sobresaliente) y 1 es la mínima (insuficiente).

Actividad física: para valorar la actividad física se utilizó el cuestionario IPAQ-A, Physical Activity Questionnaire for Adolescents (Martínez-Gómez et al., 2009). El IPAQ-A es un cuestionario sencillo que valora la actividad física que el niño realizó en los últimos siete días. Consta de cuatro grupos o partes en las que se pregunta la actividad física que los participantes hicieron durante el tiempo que estaban en el colegio, la actividad física que hicieron en casa o alrededor de ella como tareas domésticas o tareas en el jardín, la actividad física que hicieron para ir y volver de algún sitio y la actividad física que hicieron durante su tiempo libre (jugando, haciendo deporte, bailando, entrenando y/o compitiendo). A partir de este cuestionario se establecieron cuatro niveles de actividad física:

- Nivel 1 (inactivo): va en coche al colegio y apenas realiza Educación Física ni ocio activo.

Para citar este artículo utilice la siguiente referencia: Alfonso Rosa, R. Ma.;Álvarez-Barbosa, F.; Del Pozo Cruz, J. (2018). Adherencia a la dieta mediterránea, el rendimiento académico y el nivel de actividad física en edad escolar. Sportis Sci J, 4 (2), 255-268. DOI:https://doi.org/10.17979/sportis.2018.4.2.3108 


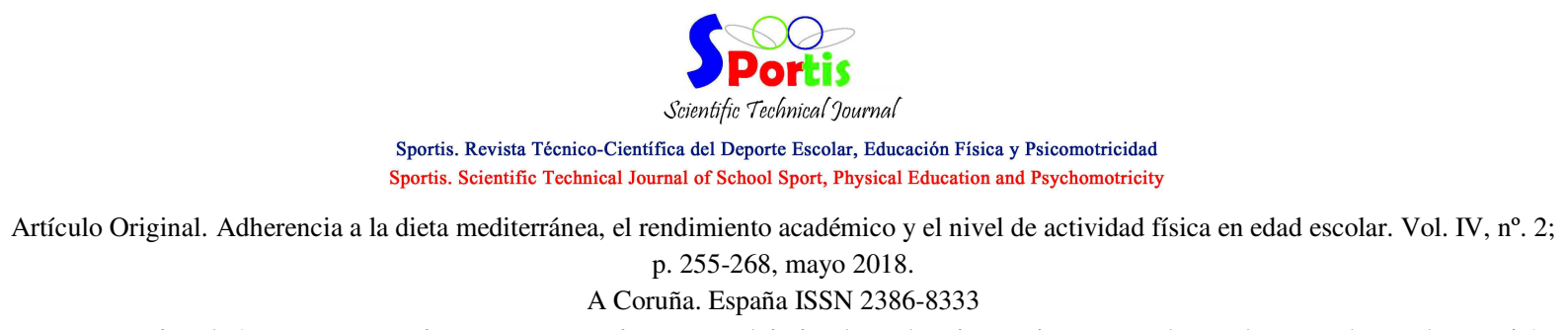

- $\quad$ Nivel 2 (poco activo): va a pie o en bici al colegio y junto a las clases de Educación Física, su actividad no llega a una hora y su ocio es de intensidad leve.

- $\quad$ Nivel 3 (moderadamente activo): va a pie o en bici al colegio, hace actividad física muy activa al menos una hora y su ocio, activo y periódico de intensidad moderada.

- $\quad$ Nivel 4 (muy activo): es aquel que va a pie o en bici al colegio, realiza las clases Educación Física y realiza deporte activo vigoroso.

Procedimiento

En primer lugar, se presentó el proyecto al director del Centro. Una vez concedido el permiso, se pasó una circular informativa a los tutores legales de los sujetos y se recogieron los respectivos consentimientos firmados. A continuación, se entregaron los diferentes cuestionarios durante el horario de tutoría. En un primer día de tutoría, fue autoadministrado el cuestionario autorregistro y el test KIDMED, y en un segundo día se autoadministró el cuestionario IPAQ-A. Por ultimo las calificaciones de las asignaturas comunes fueron reportadas por cada uno de los tutores/as de cada grupo.

\section{Análisis estadístico}

La estadística descriptiva se presentó como media y desviación estándar para las variables continuas y en términos de frecuencia y porcentajes para las variables categóricas. El nivel de asociación se estableció mediante el coeficiente de contingencia. Para todos los test, el nivel de significación se fijó en $\mathrm{p}<0,05$. Para el procesamiento y análisis de los datos se utilizó el paquete estadístico IBM-SPSS versión 22.0. (SPSS, Inc., Chicago, IL). Las figuras fueron realizadas mediante el paquete Graphpad versión 7.0 para Windows.

\section{Resultados}

A continuación, se muestran los resultados encontrados tras la aplicación de diversas pruebas estadísticas.

Para citar este artículo utilice la siguiente referencia: Alfonso Rosa, R. Mạ.;Álvarez-Barbosa, F.; Del Pozo Cruz, J. (2018). Adherencia a la dieta mediterránea, el rendimiento académico y el nivel de actividad física en edad escolar. Sportis Sci J, 4 (2), 255-268. DOI:https://doi.org/10.17979/sportis.2018.4.2.3108 
Artículo Original. Adherencia a la dieta mediterránea, el rendimiento académico y el nivel de actividad física en edad escolar. Vol. IV, $\mathrm{n}^{\circ}$. 2; p. 255-268, mayo 2018.

A Coruña. España ISSN 2386-8333

A continuación, se muestran los resultados encontrados tras la aplicación de diversas pruebas estadísticas.

La figura 1 muestra el grado de adherencia a la dieta mediterránea.
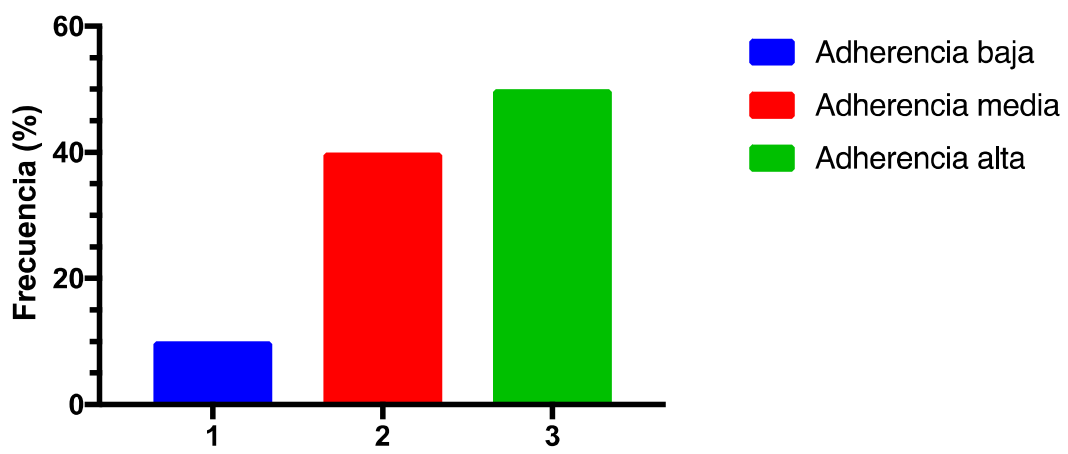

Adherencia alta

Figura 1. Grado de adherencia a la dieta mediterránea $(\mathrm{N}=50)$.

Se observa que el $50 \%$ de los sujetos tuvieron una adherencia a la dieta mediterránea óptima, frente al $10 \%$ de los sujetos que tuvieron una adherencia baja. Por otro lado, el $40 \%$ de los sujetos reflejó la necesidad de mejorar el patrón alimentario para adecuarlo al modelo mediterráneo.

En la tabla 1 se muestra la distribución según las asignaturas y la calificación obtenidas.

Tabla 1. Distribución de la muestra según asignatura y calificación obtenida $(\mathrm{N}=50)$ *

\begin{tabular}{|c|c|c|c|c|c|c|}
\hline & & Insuficiente & Suficiente & Bien & Notable & Sobresaliente \\
\hline Lengua Castellana & $\mathrm{y}$ & $2(4 \%)$ & $7(14 \%)$ & $8(16 \%)$ & $22(44 \%)$ & $11(22 \%)$ \\
\hline \multicolumn{7}{|l|}{ Literatura } \\
\hline Matemáticas & & $9(18 \%)$ & $7(14 \%)$ & $5(10 \%)$ & $19(38 \%)$ & $10(20 \%)$ \\
\hline Inglés & & $8(16 \%)$ & $13(26 \%)$ & $14(28 \%)$ & $12(24 \%)$ & $3(6 \%)$ \\
\hline Educación Física & & --- & $2(4 \%)$ & $15(30 \%)$ & $27(54 \%)$ & $6(12 \%)$ \\
\hline Educación Artística & & --- & $8(16 \%)$ & $9(18 \%)$ & $25(50 \%)$ & $8(16 \%)$ \\
\hline
\end{tabular}

Para citar este artículo utilice la siguiente referencia: Alfonso Rosa, R. M‥; Álvarez-Barbosa, F.; Del Pozo Cruz, J. (2018). Adherencia a la dieta mediterránea, el rendimiento académico y el nivel de actividad física en edad escolar. Sportis Sci J, 4 (2), 255-268. DOI:https://doi.org/10.17979/sportis.2018.4.2.3108 
Artículo Original. Adherencia a la dieta mediterránea, el rendimiento académico y el nivel de actividad física en edad escolar. Vol. IV, $\mathrm{n}^{\circ}$. 2; p. 255-268, mayo 2018.

A Coruña. España ISSN 2386-8333
CC. de la Naturaleza
$4(8 \%)$
$2(4 \%)$
$12(24 \%)$
$18(36 \%)$
$14(28 \%)$
CC. Sociales
$9(18 \%)$
$7(14 \%)$
$12(24 \%)$
$14(28 \%)$
$8(16 \%)$

* Datos presentados como frecuencias (\%)

Como se observa en la figura 2 , el $60 \%$ de los sujetos eran inactivos o muy pocos activos, frente al 25\% que fueron muy activos. Tan solo el 14\% de los sujetos realizaba la actividad física recomendada por la Organización Mundial de la Salud.

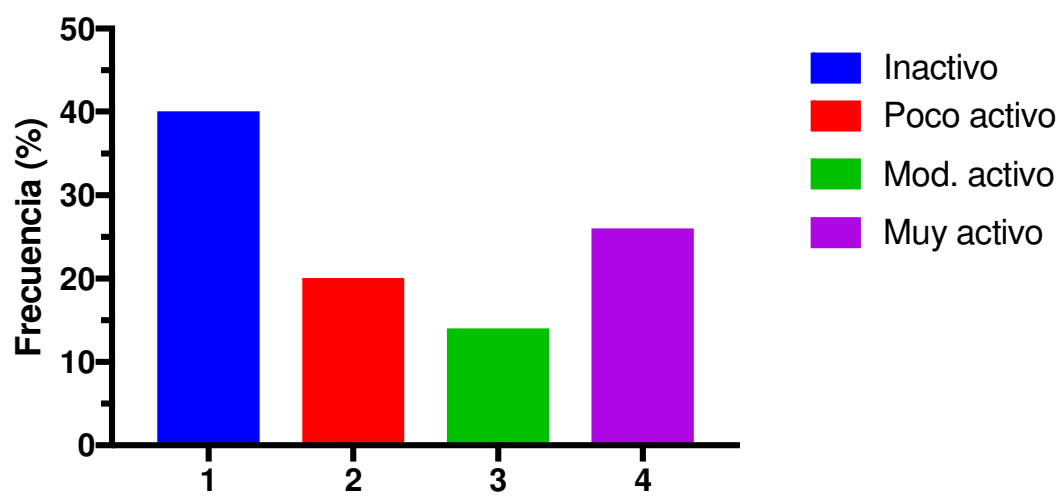

Figura 2. Nivel de práctica de actividad física de los sujetos $(\mathrm{N}=50)$.

En la figura 3 se muestra la asociación entre la adherencia a la dieta mediterránea y las calificaciones obtenidas en las diferentes asignaturas. Se observa que existe una asociación estadísticamente significativa, moderada y directamente proporcional entre la adherencia a la dieta mediterránea y las asignaturas Educación Artística (Coeficiente contingencia= ,442, $\mathrm{p}<0,05$ ), Matemáticas (Coeficiente contingencia $=, 484, \mathrm{p}<0,05)$ y Ciencias Sociales (Coeficiente contingencia $=, 490, \mathrm{p}<0,05)$.

Para citar este artículo utilice la siguiente referencia: Alfonso Rosa, R. M‥; Álvarez-Barbosa, F.; Del Pozo Cruz, J. (2018). Adherencia a la dieta mediterránea, el rendimiento académico y el nivel de actividad física en edad escolar. Sportis Sci J, 4 (2), 255-268. DOI:https://doi.org/10.17979/sportis.2018.4.2.3108 
Artículo Original. Adherencia a la dieta mediterránea, el rendimiento académico y el nivel de actividad física en edad escolar. Vol. IV, ${ }^{\circ}$. 2; p. 255-268, mayo 2018.

A Coruña. España ISSN 2386-8333
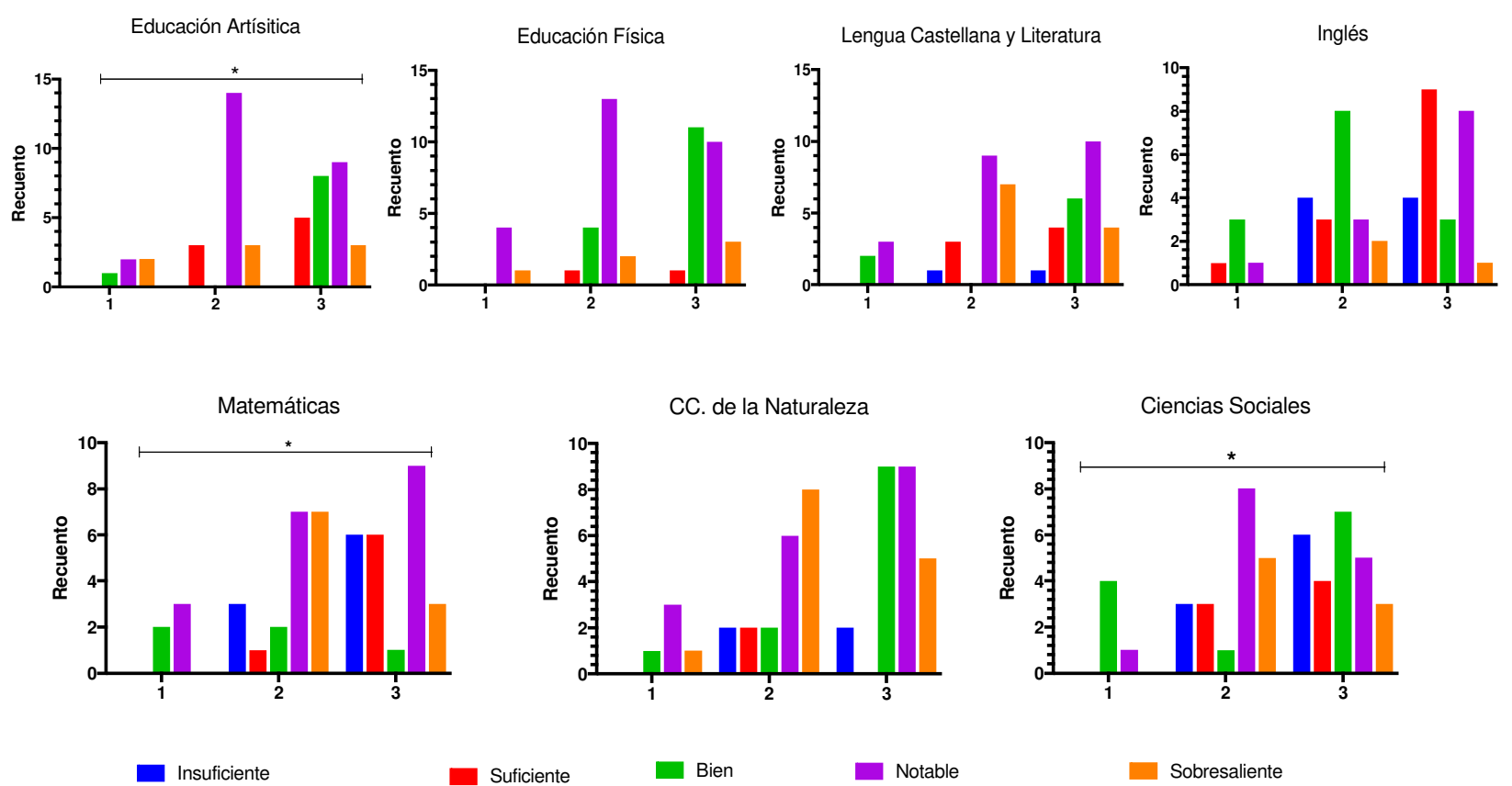

Figura 3. Asociación entre la adherencia a la dieta mediterránea y las calificaciones $(\mathrm{N}=50)$

1=Adherencia baja; $2=$ Adherencia media; $3=$ Adherencia alta $* p<0,05$

Tabla 2. Asociación entre la adherencia a la dieta mediterránea y el nivel de actividad física ( $\mathrm{N}=50)$

Inactivo Poco activo Mod. activo Muy activo

\begin{tabular}{lccccc}
\hline Adherencia baja & 2 & 1 & 1 & 1 & \\
Adherencia media & 9 & 3 & 2 & 6 &, 970 \\
Adherencia alta & 9 & 6 & 4 & 6
\end{tabular}

${ }^{*} p<0,05$

Para citar este artículo utilice la siguiente referencia: Alfonso Rosa, R. M‥; Álvarez-Barbosa, F.; Del Pozo Cruz, J. (2018). Adherencia a la dieta mediterránea, el rendimiento académico y el nivel de actividad física en edad escolar. Sportis Sci J, 4 (2), 255-268. DOI:https://doi.org/10.17979/sportis.2018.4.2.3108 


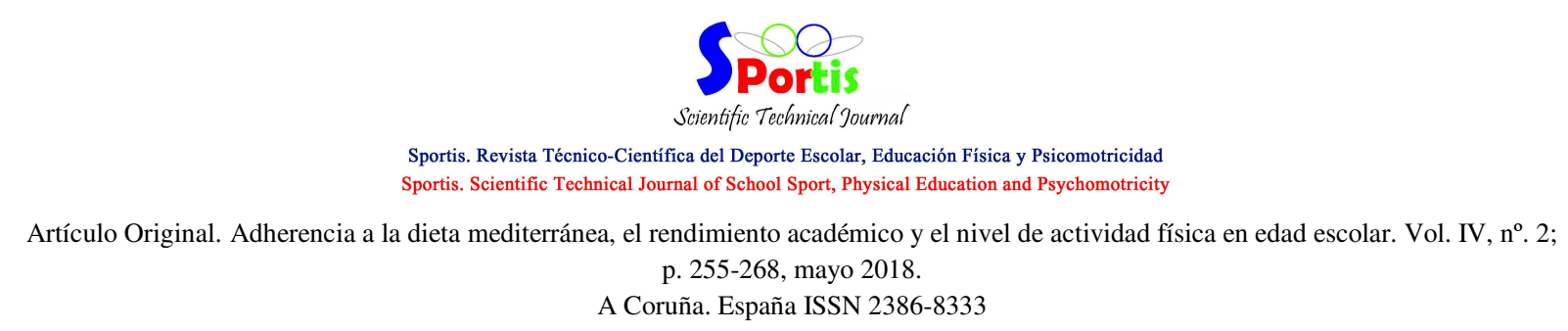

\section{Discusión y conclusiones}

El objetivo del estudio fue describir y establecer las posibles relaciones entre la adherencia a la dieta mediterránea, rendimiento académico y nivel de actividad física en alumnos de Educación Primaria pertenecientes a un centro de la provincia de Sevilla.

En cuanto a la adherencia a la dieta mediterránea se observa que la mitad de los sujetos (50\%) presentaron una adherencia a la dieta mediterránea media o baja y, por tanto, necesitaban mejorar su patrón alimentario para adecuarlo al prototipo mediterráneo. Estos resultados van en la línea de los obtenidos en el estudio EnKid, estudio pionero en el análisis de la adherencia de la dieta mediterránea a nivel nacional (Serra-Majem, Trichopoulou, et al., 2004). Sin embargo, comparando nuestros datos con los valores obtenidos por Grao-Cruces et al., (2013) se observa que estos fueron inferiores, donde los jóvenes reflejaron una alta adherencia a la dieta mediterránea $(30,9 \%)$ frente al $69,1 \%$ de sujetos que mostraba una adherencia a la dieta mediterránea media-baja.

Respecto al nivel de actividad física, se observa que el 60\% de los sujetos son inactivos o muy pocos activos, frente al $25 \%$ que son muy activos. Tan solo el $14 \%$ de los sujetos realizaba la actividad física recomendada por la Organización Mundial de la Salud (2017).

En cuanto al rendimiento académico, los resultados obtenidos en nuestro estudio son similares a los reportados por Oliver (2015) en las asignaturas de Educación Física y Educación Artística (obtienen una alta calificación) y Matemáticas y Ciencias Sociales como las asignaturas con menor calificación.

Por otro lado, atendiendo a la asociación entre la adherencia a la dieta mediterránea y el nivel de actividad física nuestro estudio mostró que no existía asociación. Estos resultados son contradictorios con los encontrados por Grao-Cruces et al., (2013) donde se observó que los adolescentes con una alta adherencia a la dieta mediterránea eran más activos que aquellos que necesitaban mejorar el patrón dietético adaptándolo al mediterráneo. Aunque no siempre los niños más activos se inclinan hacia una alimentación más saludable (Ottevaere et al., 2011), sí que se observa en anteriores trabajos dedicados al estudio de la adherencia a la dieta mediterránea y la actividad física, una mayor adherencia a dicha dieta entre los sujetos con un

Para citar este artículo utilice la siguiente referencia: Alfonso Rosa, R. Mạ.;Álvarez-Barbosa, F.; Del Pozo Cruz, J. (2018). Adherencia a la dieta mediterránea, el rendimiento académico y el nivel de actividad física en edad escolar. Sportis Sci J, 4 (2), 255-268. DOI:https://doi.org/10.17979/sportis.2018.4.2.3108 


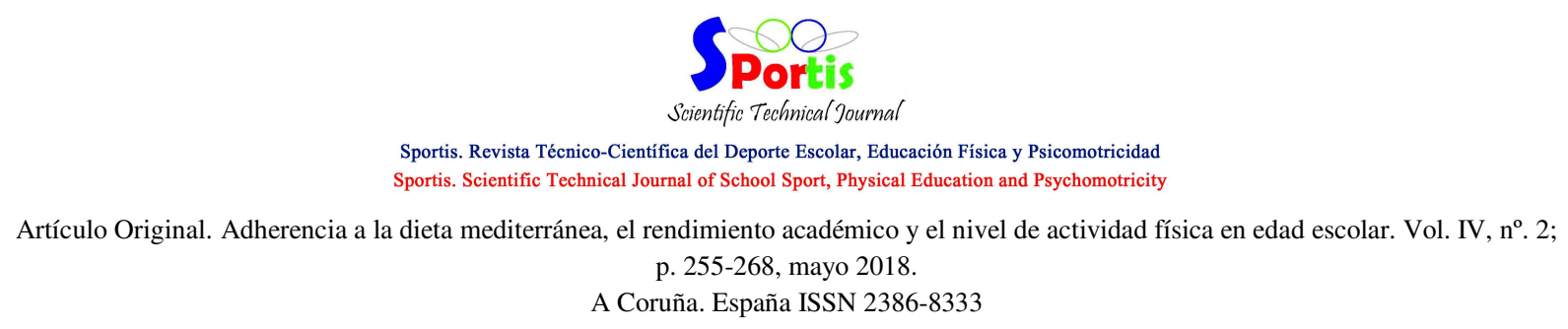

mayor nivel de actividad física (Farajian et al., 2011; Schröder, Mendez, Ribas-Barba, Covas, \& Serra-Majem, 2010).

Por último, cabe destacar la asociación entre adherencia a la dieta mediterránea y el rendimiento académico. Esta asociación fue estadísticamente significativa, moderada y directamente proporcional entre la adherencia a la dieta mediterránea y las asignaturas de Educación Artística, Matemáticas y Ciencias Sociales. Estos resultados son consistentes con los reportados por Shi, Tubb, Fingers, Chen, \& Caffrey (2013), los cuales concluyeron que los niños que toman comidas más saludables y hacen ejercicio muestran una mejor salud y menos problemas tanto de comportamiento como académicos. Además, indican que la incidencia de problemas académicos y de conducta en niños sedentarios fue entre un 10-12\% mayor que la de los sujetos activos físicamente. A su vez, Álvarez-Bueno et al., (2017) muestran que la actividad física, en concreto la Educación Física, mejora el comportamiento en el aula y beneficia varios aspectos del rendimiento académico, especialmente las habilidades relacionadas con las matemáticas y la lectura.

Por tanto, podemos concluir que el $50 \%$ de los sujetos tiene que mejorar su patrón alimentario, que tan solo el $14 \%$ realiza la actividad física recomendada y que existió asociación entre la adherencia a la dieta mediterránea y las calificaciones obtenidas en Educación Artística, Matemáticas y Ciencias Sociales.

Sin embargo, como cualquier investigación, ésta también tiene limitaciones que requieren que se siga investigando para obtener datos que contrasten los resultados obtenidos (análisis más global de las materias académicas, sexo, niveles de actividad física, entre otros).

Por tanto, futuras investigaciones podríamos destacar: aumentar la muestra para indagar aún más en los ámbitos estudiados, incluir el rol del profesor, padre, madre o tutor como factor influyente en la adquisición de hábitos saludables, análisis de las variables por sexo, cursos...

\section{Referencias bibliográficas}

1. AECOSAN. (2016). ALADINO 2015. http://doi.org/690-16-010-3

2. Álvarez-Bueno, C., Pesce, C., Cavero-Redondo, I., Sánchez-López, M., GarridoMiguel, M., \& Martínez-Vizcaíno, V. (2017). Academic Achievement and Physical Para citar este artículo utilice la siguiente referencia: Alfonso Rosa, R. Ma.; Álvarez-Barbosa, F.; Del Pozo Cruz, J. (2018). Adherencia a la dieta mediterránea, el rendimiento académico y el nivel de actividad física en edad escolar. Sportis Sci J, 4 (2), 255-268. DOI:https://doi.org/10.17979/sportis.2018.4.2.3108 
Artículo Original. Adherencia a la dieta mediterránea, el rendimiento académico y el nivel de actividad física en edad escolar. Vol. IV, nº 2 ; p. 255-268, mayo 2018.

A Coruña. España ISSN 2386-8333

Activity: A Meta-analysis. Pediatrics, 140(6), e20171498. http://doi.org/10.1542/peds.2017-1498

3. Bellisle, F. (2004). Effects of diet on behaviour and cognition in children. British Journal of Nutrition, 92(S2), S227. http://doi.org/10.1079/BJN20041171

4. Bulló, M., Lamuela-Raventós, R., \& Salas-Salvadó, J. (2011). Mediterranean diet and oxidation: nuts and olive oil as important sources of fat and antioxidants. Current Topics in Medicinal Chemistry, 11(14), 1797-810. Retrieved from http://www.ncbi.nlm.nih.gov/pubmed/21506929

5. Castro-Quezada, I., Román-Viñas, B., \& Serra-Majem, L. (2014). The Mediterranean diet and nutritional adequacy: a review. Nutrients, 6(1), 231-48. http://doi.org/10.3390/nu6010231

6. Costarelli, V., Koretsi, E., \& Georgitsogianni, E. (2013). Health-related quality of life of Greek adolescents: the role of the Mediterranean diet. Quality of Life Research, 22(5), 951-956. http://doi.org/10.1007/s11136-012-0219-2

7. Donini, L. M., Serra-Majem, L., Bulló, M., Gil, Á., \& Salas-Salvadó, J. (2015). The Mediterranean diet: culture, health and science. British Journal of Nutrition, 113(S2), S1-S3. http://doi.org/10.1017/S0007114515001087

8. Estruch, R., Ros, E., Salas-Salvadó, J., Covas, M.-I., Corella, D., Arós, F., ... PREDIMED Study Investigators. (2013). Primary Prevention of Cardiovascular Disease with a Mediterranean Diet. New England Journal of Medicine, 368(14), 1279-1290. http://doi.org/10.1056/NEJMoa1200303

9. Farajian, P., Risvas, G., Karasouli, K., Pounis, G. D., Kastorini, C. M., Panagiotakos, D. B., \& Zampelas, A. (2011). Very high childhood obesity prevalence and low adherence rates to the Mediterranean diet in Greek children: The GRECO study. Atherosclerosis, 217(2), 525-530. http://doi.org/10.1016/j.atherosclerosis.2011.04.003

10. Grao-Cruces, A., Nuviala, A., Fernández-Martínez, A., Porcel-Gálvez, A.-M., MoralGarcía, J.-E., \& Martínez-López, E. J. (2013). Adherencia a la dieta mediterránea en adolescentes rurales y urbanos del sur de España, satisfacción con la vida, antropometría y actividades físicas y sedentarias. Nutrición Hospitalaria, 28(4), 1129 1135. http://doi.org/10.3305/nh.2013.28.4.6486

Para citar este artículo utilice la siguiente referencia: Alfonso Rosa, R. Mạ.;Álvarez-Barbosa, F.; Del Pozo Cruz, J. (2018). Adherencia a la dieta mediterránea, el rendimiento académico y el nivel de actividad física en edad escolar. Sportis Sci J, 4 (2), 255-268. DOI:https://doi.org/10.17979/sportis.2018.4.2.3108 


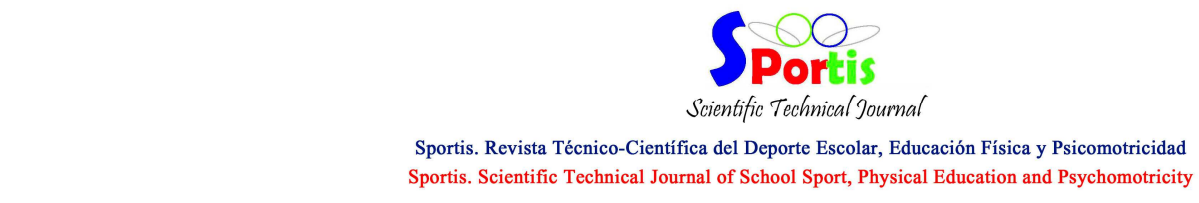

Artículo Original. Adherencia a la dieta mediterránea, el rendimiento académico y el nivel de actividad física en edad escolar. Vol. IV, $\mathrm{n}^{\circ}$. 2; p. 255-268, mayo 2018.

A Coruña. España ISSN 2386-8333

11. Karlén, J., Lowert, Y., Chatziarsenis, M., Fälth-Magnusson, K., \& Faresjö, T. (n.d.). Are children from Crete abandoning a Mediterranean diet? Rural and Remote Health, 8(4), 1034. Retrieved from http://www.ncbi.nlm.nih.gov/pubmed/19014272

12. Lazarou, C., \& Kalavana, T. (2009). Urbanization influences dietary habits of Cypriot children: the CYKIDS study. International Journal of Public Health, 54(2), 69-77. http://doi.org/10.1007/s00038-009-8054-0

13. Macias M, A. I., Gordillo S, L. G., \& Camacho R, E. J. (2012). Hábitos alimentarios de niños en edad escolar y el papel de la educación para la salud. Revista Chilena de Nutrición, 39(3), 40-43. http://doi.org/10.4067/S0717-75182012000300006

14. Martínez-Gómez, D., Martínez-de-Haro, V., Pozo, T., Welk, G. J., Villagra, A., Calle, M. E., ... Veiga, O. L. (2009). Reliability and Validity of the PAQ-A Questionnaire to Assess Physical La actividad física se define como actividad física ha sido identificada como un agente releva. Rev Esp Salud Pública, 83(3), 427-439. http://doi.org/10.1590/S1135-57272009000300008

15. Molinero, O., Castro-Piñero, J., Ruiz, J. R., González Montesinos, J. L., Mora, J., \& Márquez, S. (2010). Conductas de salud en escolares de la provincia de C??diz. Nutricion Hospitalaria, 25(2), 280-289. http://doi.org/10.3305/nh.2010.25.2.4579

16. Nadtochiy, S. M., \& Redman, E. K. (2011). Mediterranean diet and cardioprotection: The role of nitrite, polyunsaturated fatty acids, and polyphenols. Nutrition, 27(7-8), 733-744. http://doi.org/10.1016/j.nut.2010.12.006

17. OMS. (2014). OMS | Datos y cifras sobre obesidad infantil. WHO. World Health Organization. Retrieved from http://www.who.int/end-childhood-obesity/facts/es/

18. Ottevaere, C., Huybrechts, I., De Bourdeaudhuij, I., Sjöström, M., Ruiz, J. R., Ortega, F. B., ... De Henauw, S. (2011). Comparison of the IPAQ-A and Actigraph in relation to VO 2 max among European adolescents: The HELENA study. Journal of Science and Medicine in Sport, 14, 317-324. http://doi.org/10.1016/j.jsams.2011.02.008

19. Owen, N., Sparling, P. B., Healy, G. N., Dunstan, D. W., \& Matthews, C. E. (2010). Sedentary Behavior: Emerging Evidence for a New Health Risk. Mayo Clinic Proceedings, 85(12), 1138-1141. http://doi.org/10.4065/mcp.2010.0444

20. Palmer, R. H. C., Young, S. E., Hopfer, C. J., Corley, R. P., Stallings, M. C., Crowley, T. J., \& Hewitt, J. K. (2009). Developmental epidemiology of drug use and abuse in Para citar este artículo utilice la siguiente referencia: Alfonso Rosa, R. M‥; Álvarez-Barbosa, F.; Del Pozo Cruz, J. (2018). Adherencia a la dieta mediterránea, el rendimiento académico y el nivel de actividad física en edad escolar. Sportis Sci J, 4 (2), 255-268. DOI:https://doi.org/10.17979/sportis.2018.4.2.3108 


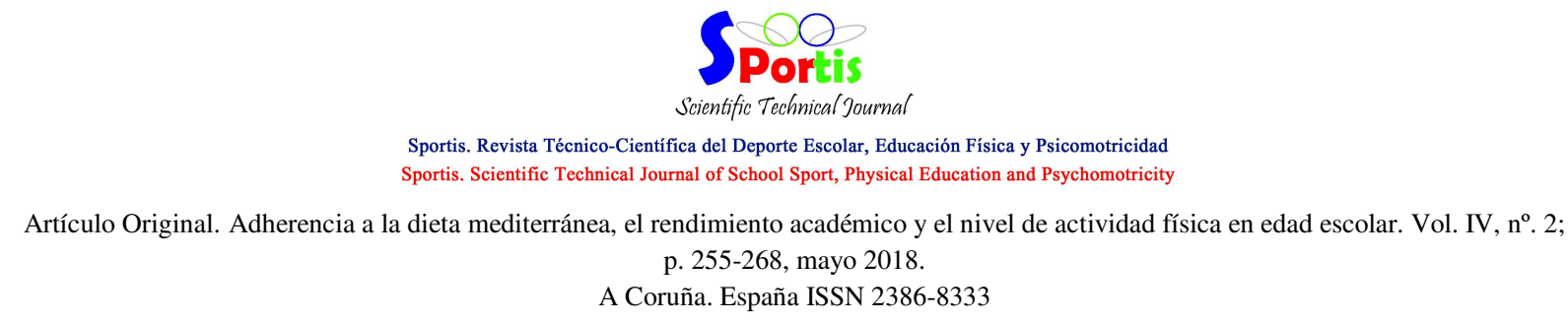

adolescence and young adulthood: Evidence of generalized risk. Drug and Alcohol Dependence, 102(1-3), 78-87. http://doi.org/10.1016/j.drugalcdep.2009.01.012

21. Schröder, H., Mendez, M. A., Ribas-Barba, L., Covas, M.-I., \& Serra-Majem, L. (2010). Mediterranean diet and waist circumference in a representative national sample of young Spaniards. International Journal of Pediatric Obesity, 5(6), 516-519. http://doi.org/10.3109/17477161003777417

22. Serra-Majem, L., Ribas, L., Ngo, J., Ortega, R. M., García, A., Pérez-Rodrigo, C., \& Aranceta, J. (2004). Food, youth and the Mediterranean diet in Spain. Development of KIDMED, Mediterranean Diet Quality Index in children and adolescents. Public Health Nutrition, 7(7), 931-5. Retrieved from http://www.ncbi.nlm.nih.gov/pubmed/15482620

23. Serra-Majem, L., Trichopoulou, A., de la Cruz, J. N., Cervera, P., Álvarez, A. G., La Vecchia, C., ... Trichopoulos, D. (2004). Does the definition of the Mediterranean diet need to be updated? Public Health Nutrition, 7(7), 927-929. http://doi.org/10.1079/PHN2004564

24. Shi, X., Tubb, L., Fingers, S. T., Chen, S., \& Caffrey, J. L. (2013). Associations of Physical Activity and Dietary Behaviors With Children's Health and Academic Problems. Journal of School Health, 83(1), 1-7. http://doi.org/10.1111/j.17461561.2012.00740.x

25. Tognon, G., Hebestreit, A., Lanfer, A., Moreno, L. A., Pala, V., Siani, A., ... Lissner, L. (2014). Mediterranean diet, overweight and body composition in children from eight European countries: Cross-sectional and prospective results from the IDEFICS study. Nutrition, Metabolism and Cardiovascular Diseases, 24(2), 205-213. http://doi.org/10.1016/j.numecd.2013.04.013

Para citar este artículo utilice la siguiente referencia: Alfonso Rosa, R. Mạ.;Álvarez-Barbosa, F.; Del Pozo Cruz, J. (2018). Adherencia a la dieta mediterránea, el rendimiento académico y el nivel de actividad física en edad escolar. Sportis Sci J, 4 (2), 255-268. DOI:https://doi.org/10.17979/sportis.2018.4.2.3108 